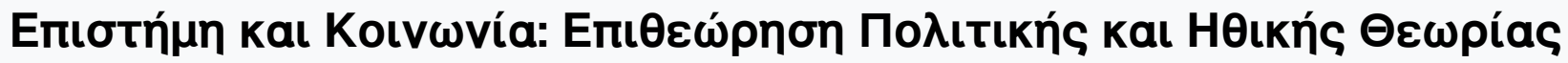

Tóp. 1 (1998)

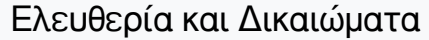

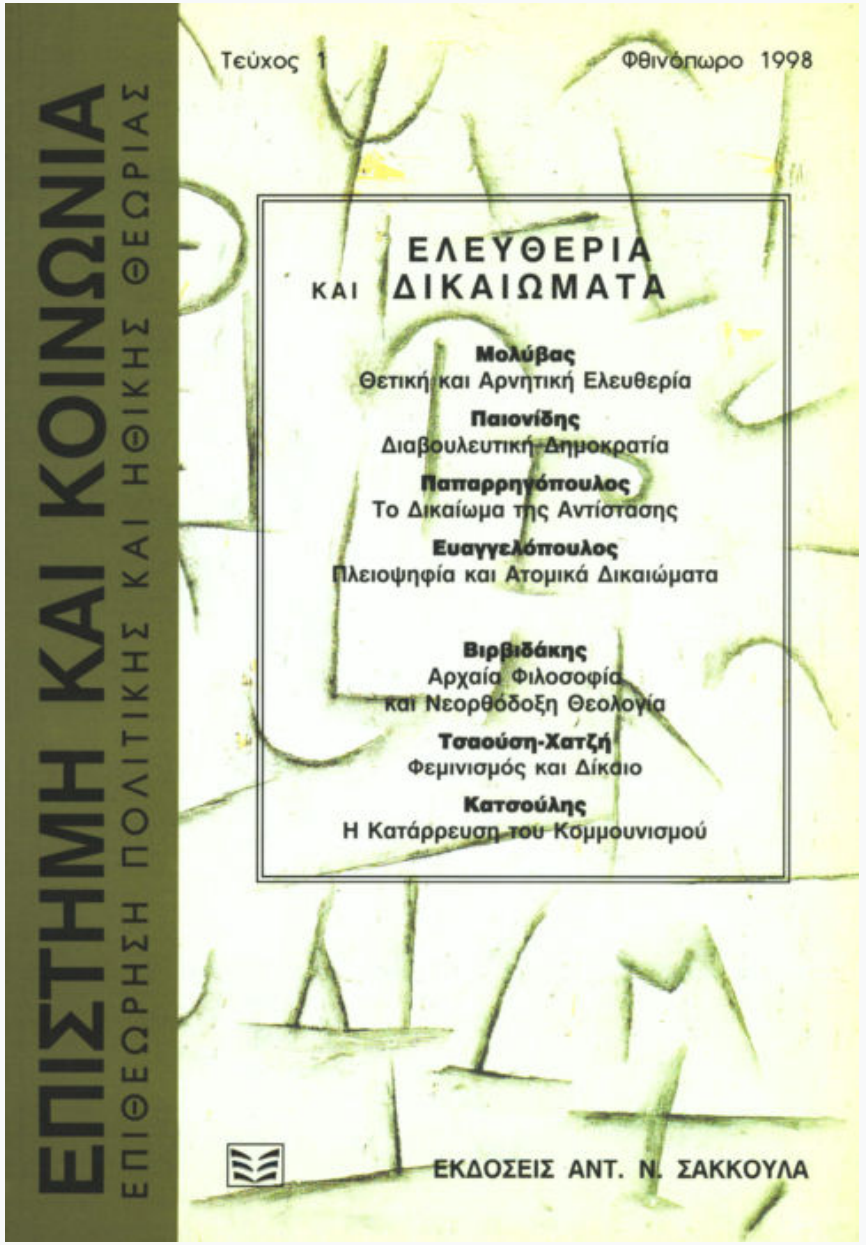

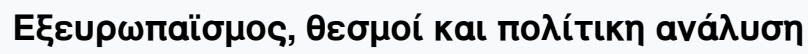

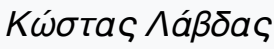

doi: $\underline{10.12681 / \text { sas. } 688}$

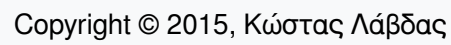

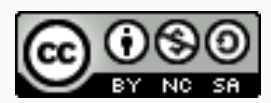

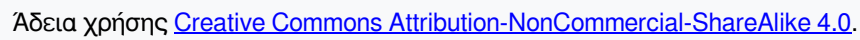

\section{Bıß入ıоррачıкń avaџopá:}

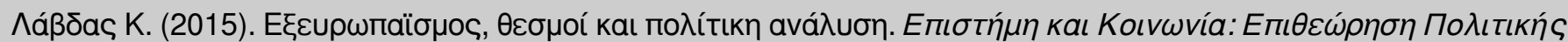

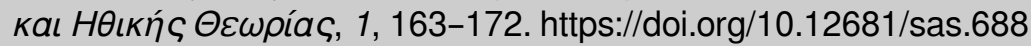




\title{
ВIВАIОГРАФІКО АОКIMIO
}

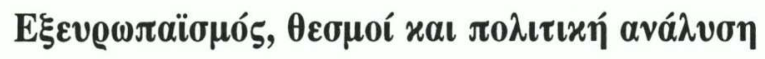

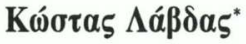

Andersen, S. \& Eliassen, K. eds. (1993). Making Policy in Europe: The Europeification of National Policy-Making. London: Sage.

Carlsnaes, W. \& Smith, S. eds. (1994). European Foreign Policy: The EC and Changing Perspectives in Europe. London: Sage.

Greenwood, J. (1997). Representing Interests in the European Union. London: Macmillan.

Kassim, H. \& Menon, A. eds. (1996). The European Union and National Industrial Policy. London: Routlegde.

Marks, G., Scharpf, F.W., Schmitter, P.C., \& Streeck, W. (1996). Governance in the European Union. London: Sage.

Meny, Y. et al. (1997). Adjusting to Europe: The Impact of the European Union on National Institutions and Policies. London: Routledge.

Rometsch, D. \& Wessels, W. eds. (1996). The European Union and Member States: Towards Institutional Fusion? Manchester: Manchester University Press.

Schneider, H. \& Wessels, W. eds. (1994). Foederale Union: Europas Zukunft? Muenchen: Beck.

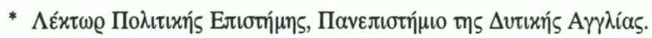




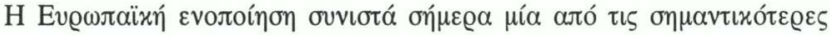

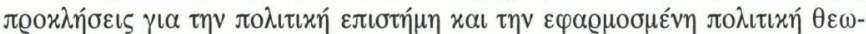

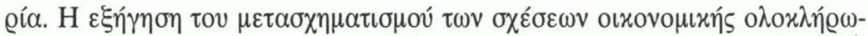

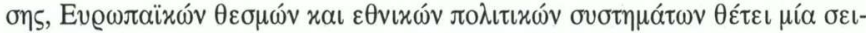

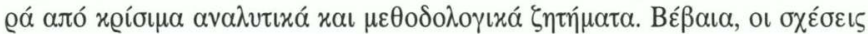

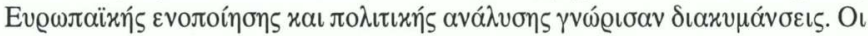

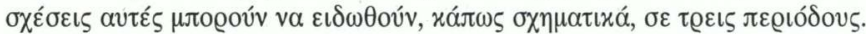

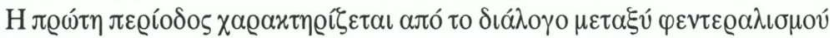

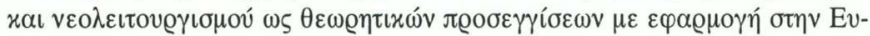

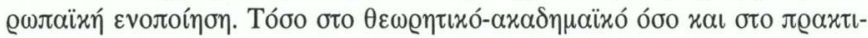

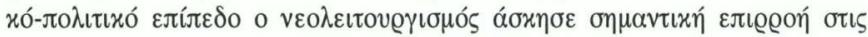

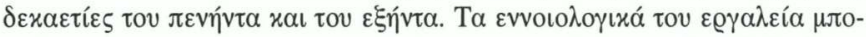

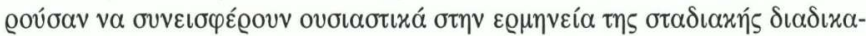

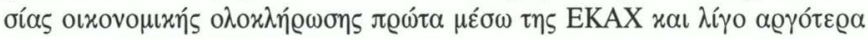

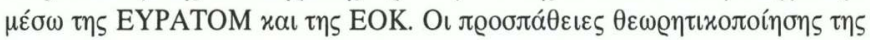

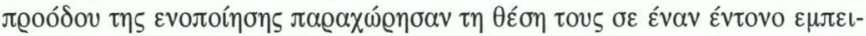

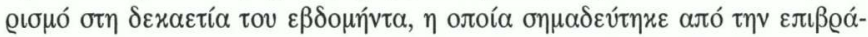

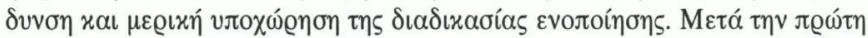

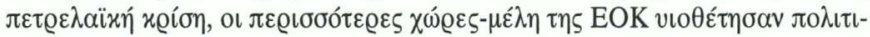

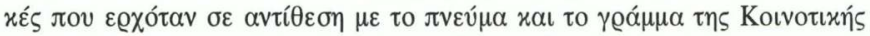

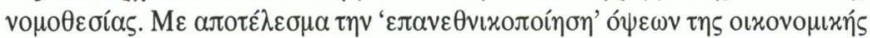

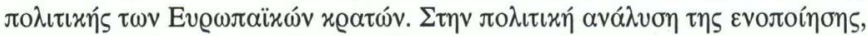

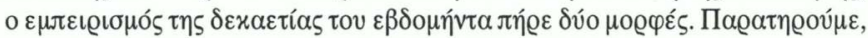

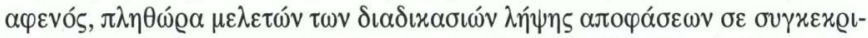

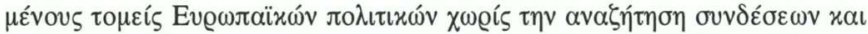

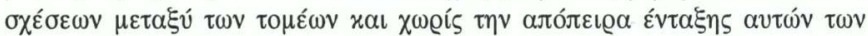

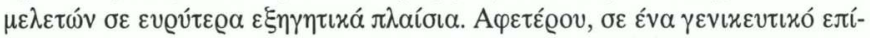

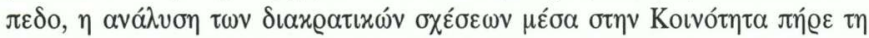

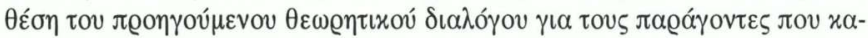

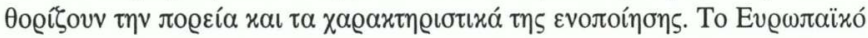

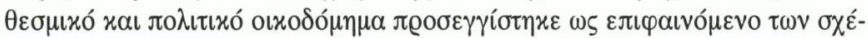

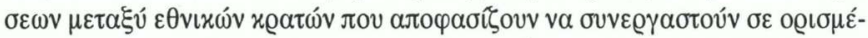

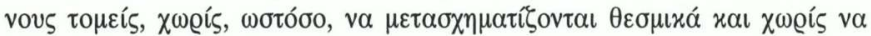

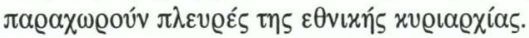

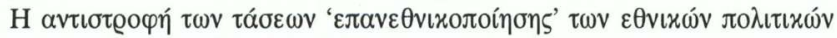

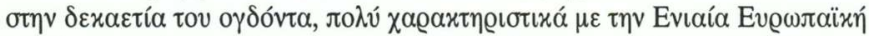

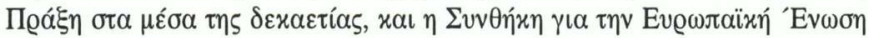




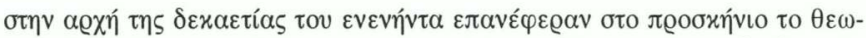

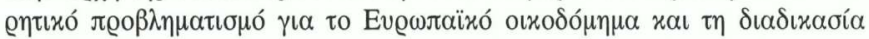

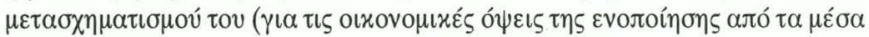

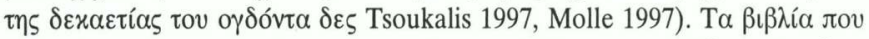

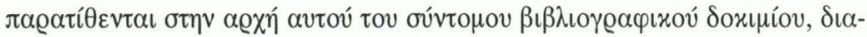

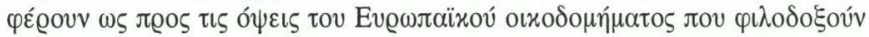

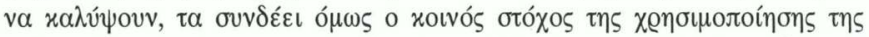

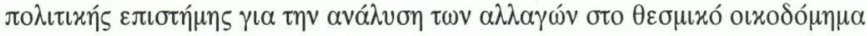

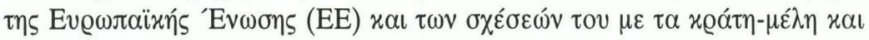

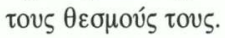

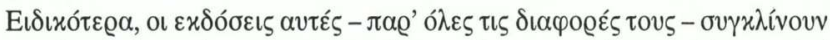

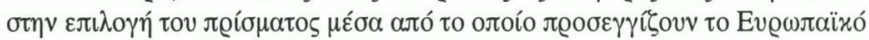

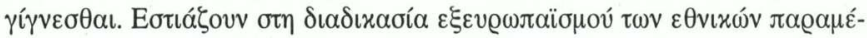

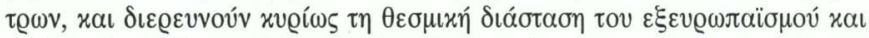

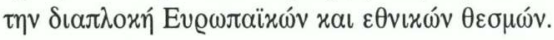

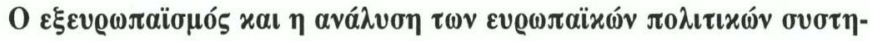 $\mu \alpha ́ \tau \omega v$.}

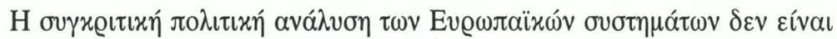

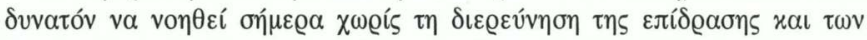

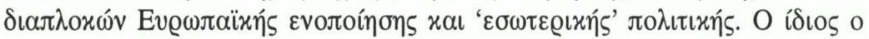

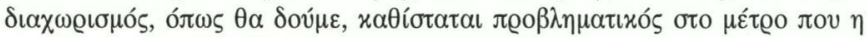

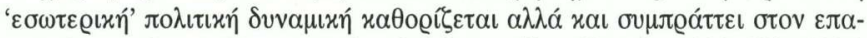

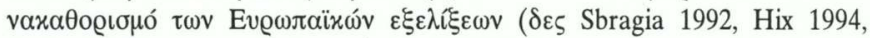

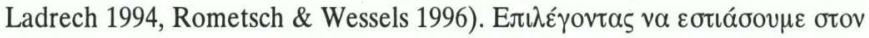

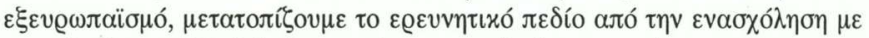

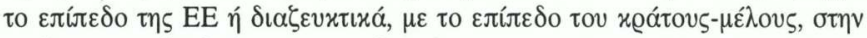

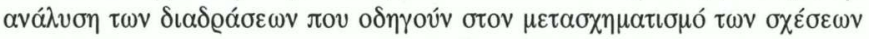

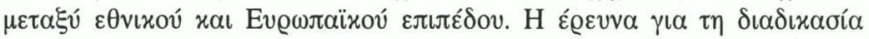

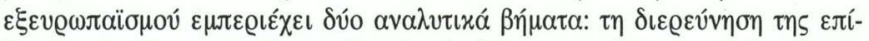

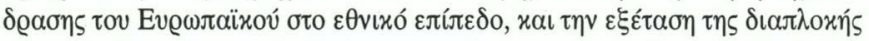

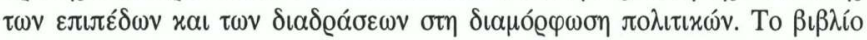
$\tau \omega v$ Marks, Scharpf, Schmitter xaı Streeck (1996) a

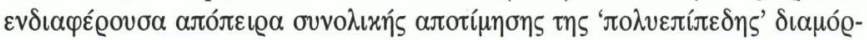

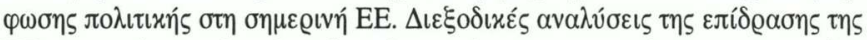

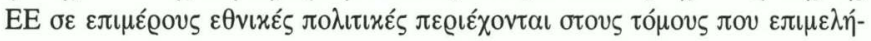




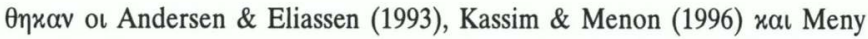

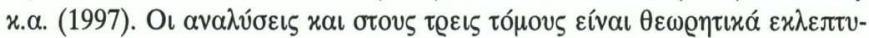

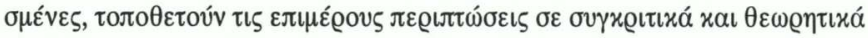

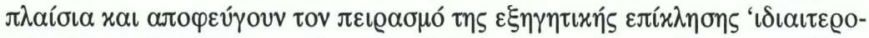

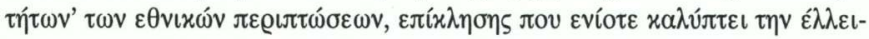

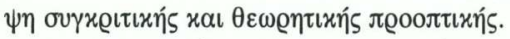

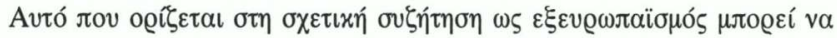

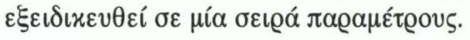

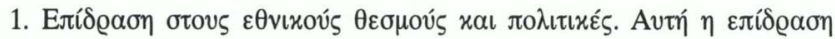

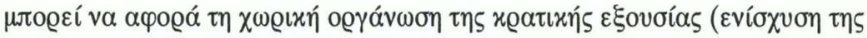

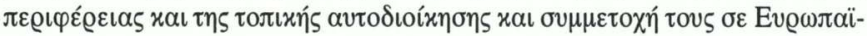

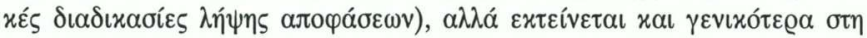

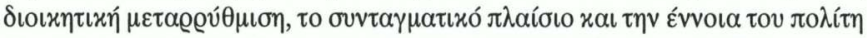

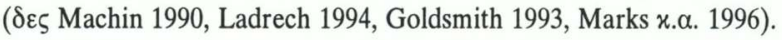

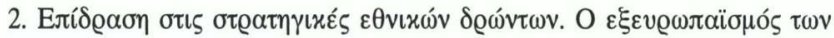

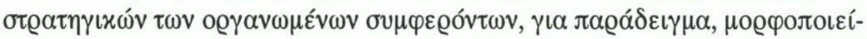

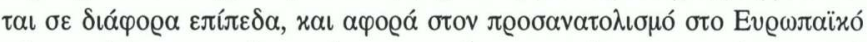

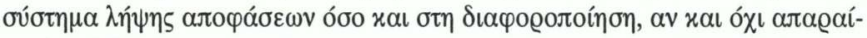

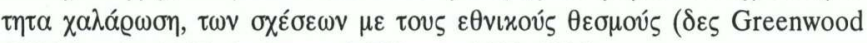
1997, Lavdas 1997, Andersen \& Eliassen 1993, 1991).

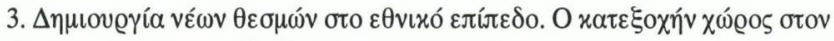

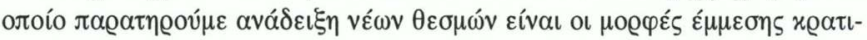

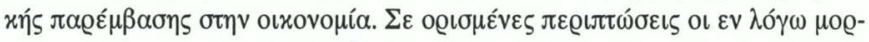

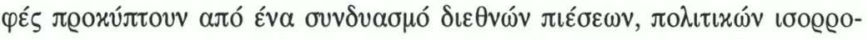

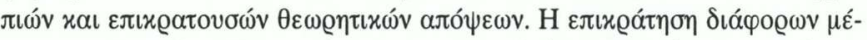

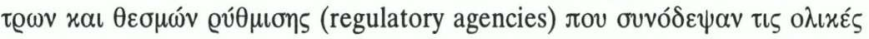

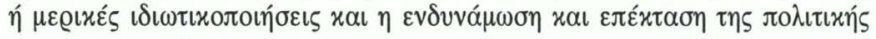

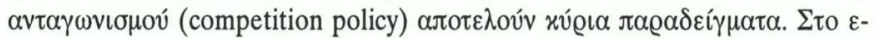

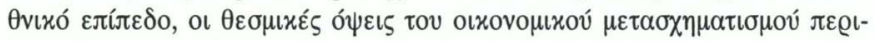

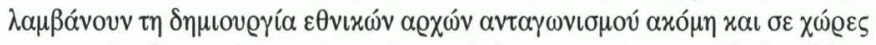

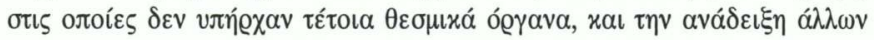

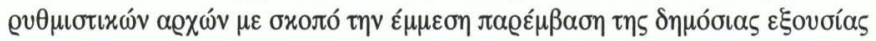

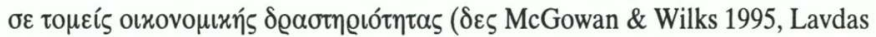
\& Mendrinou 1995, Lavdas 1996, Hancher oє Kassim \& Menon 1996, Amato

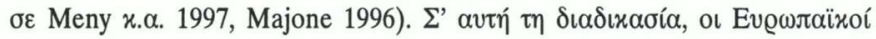

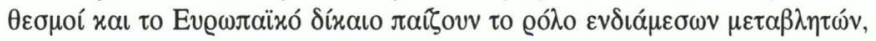




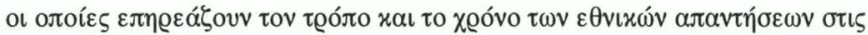

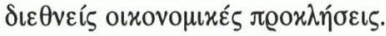

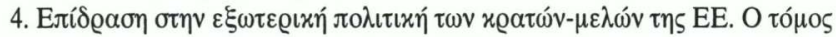

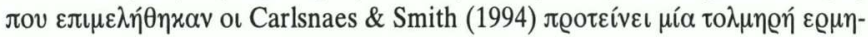

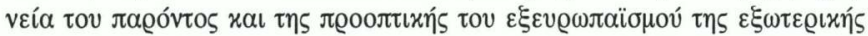

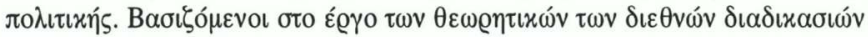

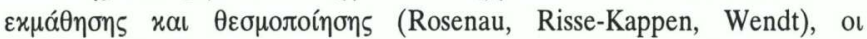

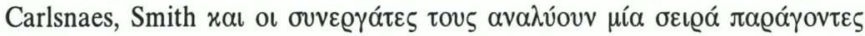

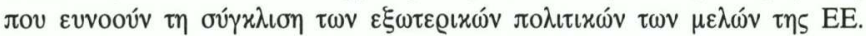

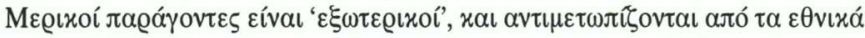

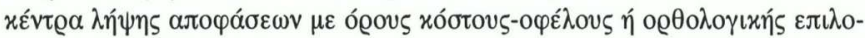

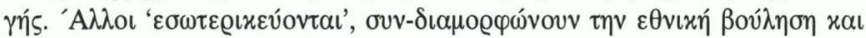

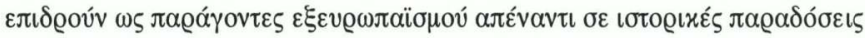

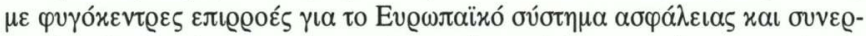

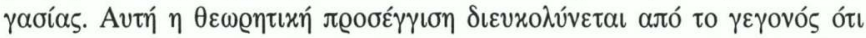

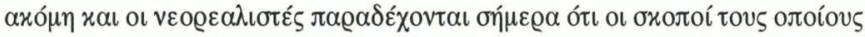

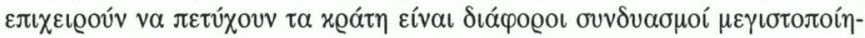

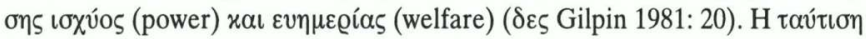

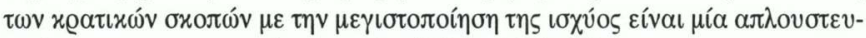

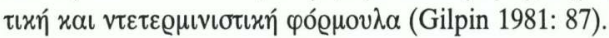

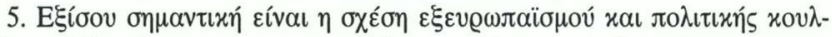

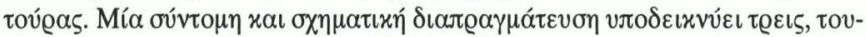

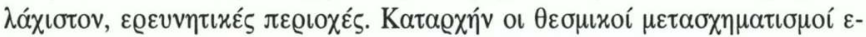

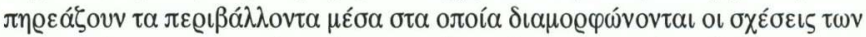

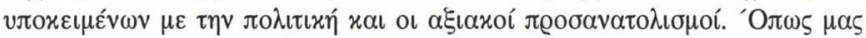

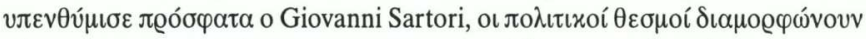

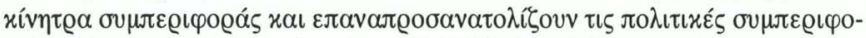

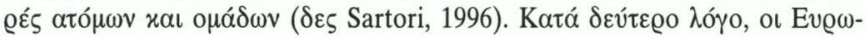

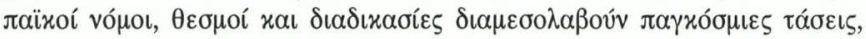

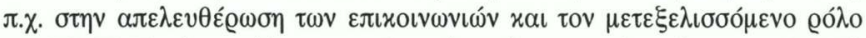

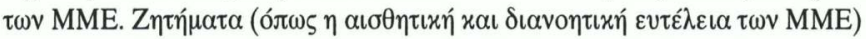

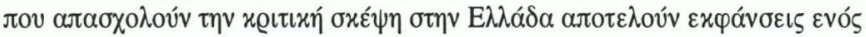

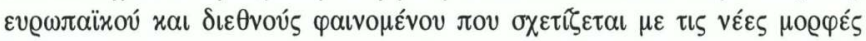

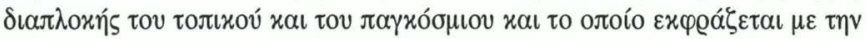

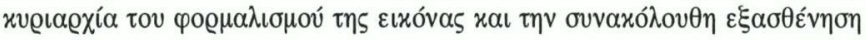

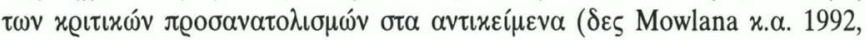

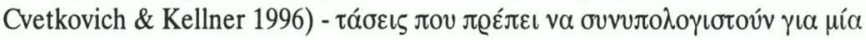




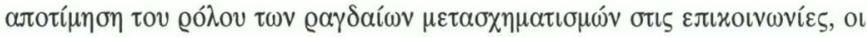

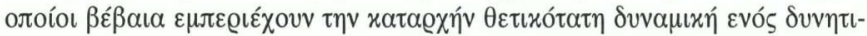

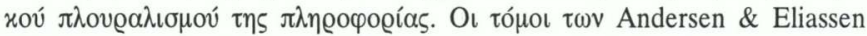

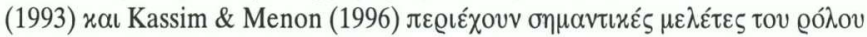

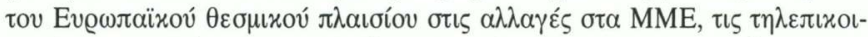

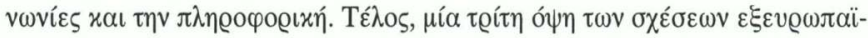

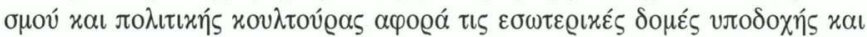

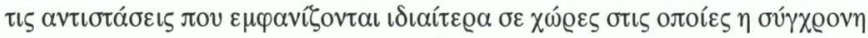

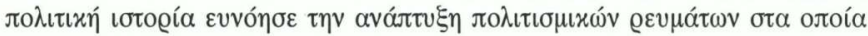

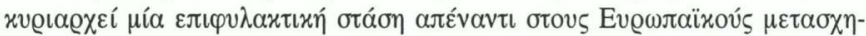

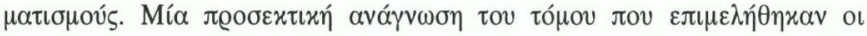

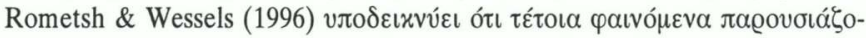

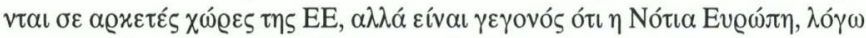

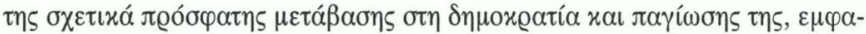

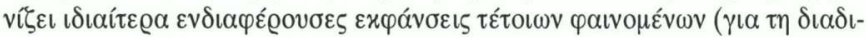

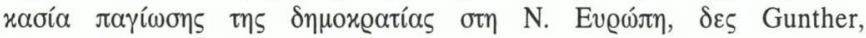
Diamandouros \& Puhle 1995).

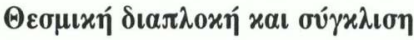

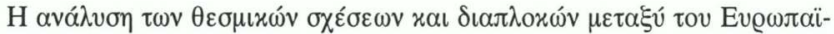

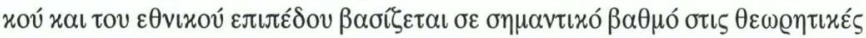

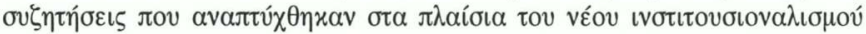

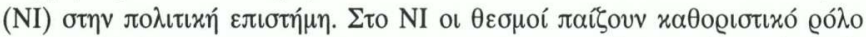

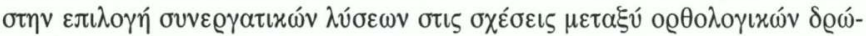

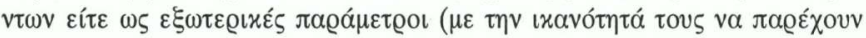

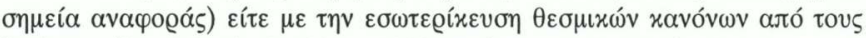

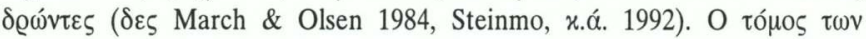

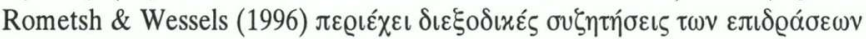

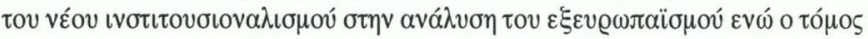

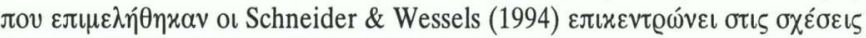

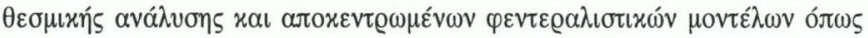

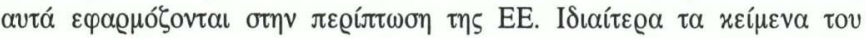

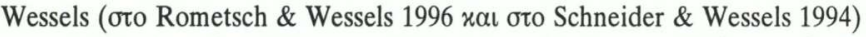

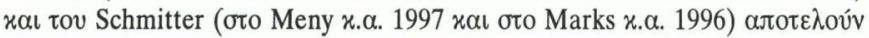

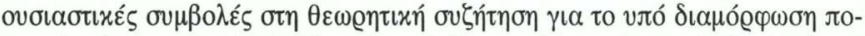

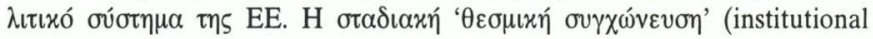




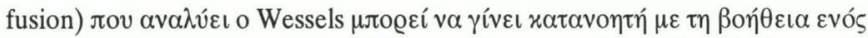

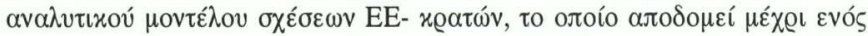

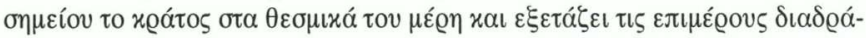

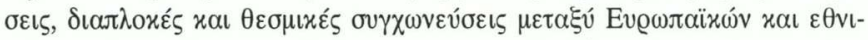

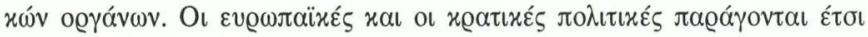

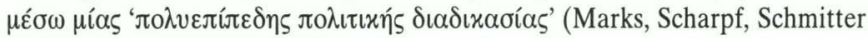

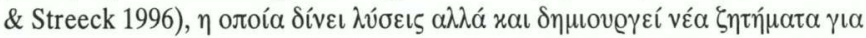

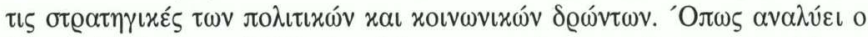

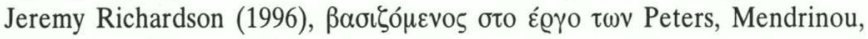

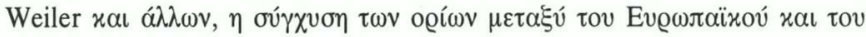

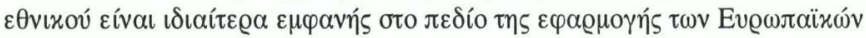

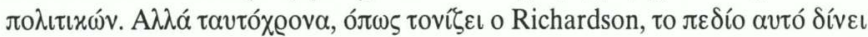

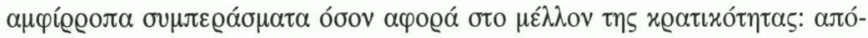

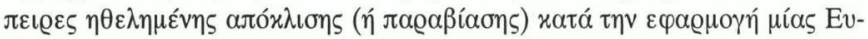

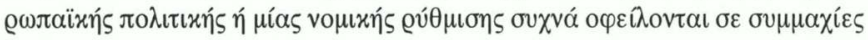

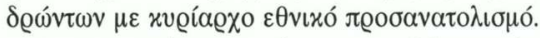

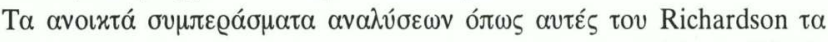

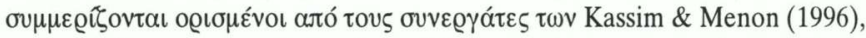

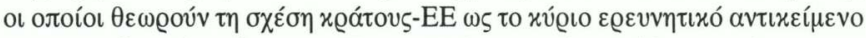

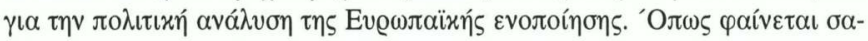

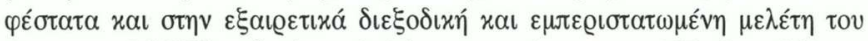

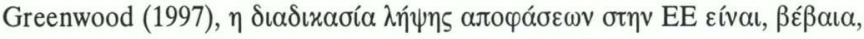

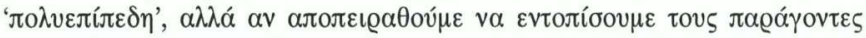

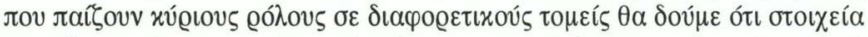

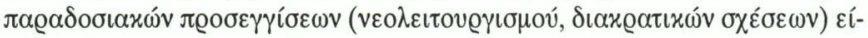

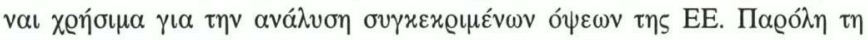

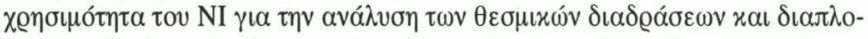

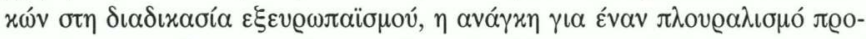

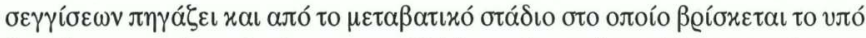

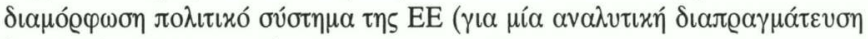
$\delta \varepsilon \zeta$ Lavdas 1997: $\chi \varepsilon \varphi .2)$.

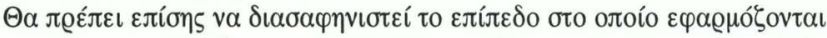

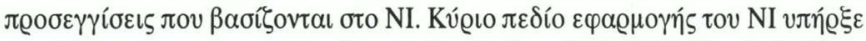

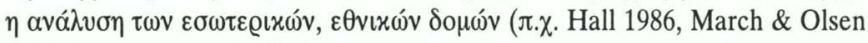

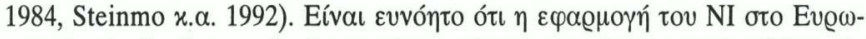

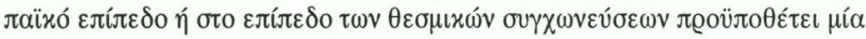

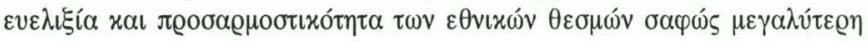




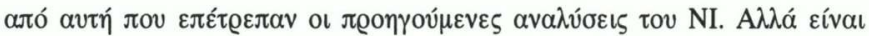

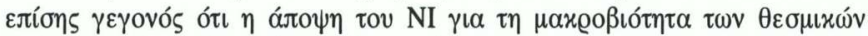

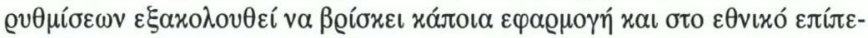

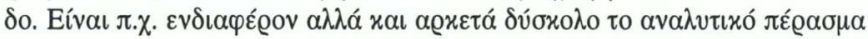

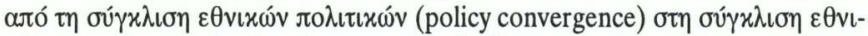

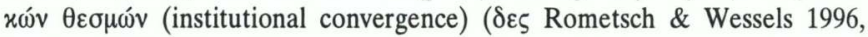

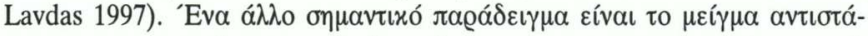

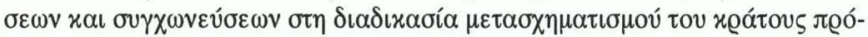

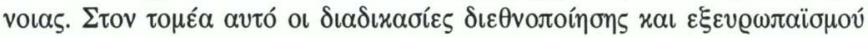

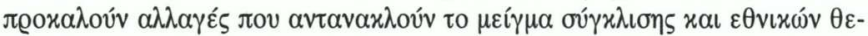

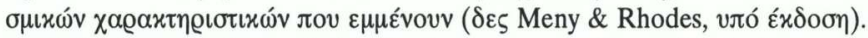

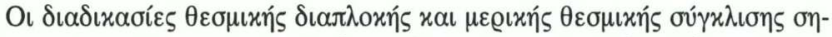

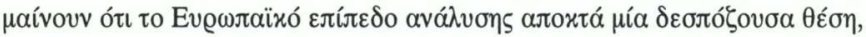

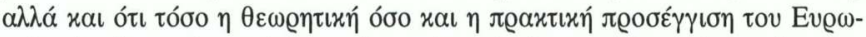

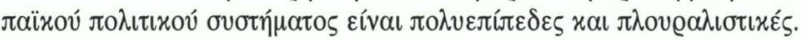

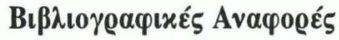

Andersen, S. \& Eliassen, K. (1991). 'European Community Lobbying', European Journal of Political Research, 20.

Cvetkovich, A. \& Kellner, D. (1996). Articulating the Global and the Local. Boulder: Westview Press.

Gilpin, R. (1981). War and Change in World Politics. Cambridge: Cambridge University Press.

Goldsmith, M. (1993). 'The Europeanization of Local Government', Urban Studies, 30.

Gunther, R., Diamandouros, P.N., \& Puhle, H.J. eds. (1995). The Politics of Democratic Consolidation: Southern Europe in Comparative Perspective. Baltimore: Johns Hopkins University Press.

Hall, P. (1986). Governing the Economy: The Politics of State Intervention in Britain and France. Oxford: Oxford University Press.

Hix, S. (1994). 'Approaches to the Study of the EC: The Challenge to Comparative Politics', West European Politics, 17.

Ladrech, R. (1994). 'Europeanization of Domestic Politics and Institutions: The Case of France', Journal of Common Market Studies, 32.

Lavdas, K. (1996). 'The Political Economy of Privatization in Southern Europe', in D. Braddon \& D. Foster, eds., Privatization. London: Ashgate. 
Lavdas, K. (1997). The Europeanization of Greece: Interest Politics and the Crises of Integration. London: Macmillan.

Lavdas, K. \& Mendrinou, M. (1995). 'Competition Policy and Institutional Politics in the European Community', European Journal of Political Research, 28.

Machin, H. (1990). 'Introduction', in P. Hall et al., Developments in French Politics. London: Macmillan.

Majone, G. ed. (1996). Regulating Europe. London: Routledge.

March, J. \& Olsen, J. (1984). 'The New Institutionalism: Organizational Factors in Political Life', American Political Science Review, 78.

McGowan, L. \& Wilks, S. (1995). 'The First Supranational Policy in the European Union: Competition Policy', European Journal of Political Research, 28.

Mendrinou, M. (1996). 'Non-Compliance and the European Commission's Role in Integration', Journal of European Public Policy, 3.

... (forthcoming). Integration and Enforcement. Oxford: Oxford University Press.

Meny, Y. \& Rhodes, M. (forthcoming). A New Social Contract? Charting the Future of European Welfare. London: Macmillan.

Molle, W. (1997). The Economics of European Integration. Third Edition. London: Ashgate.

Mowlana, H. et al. eds. (1992). Triumph of the Image. Boulder: Westview Press.

Peters, B.G. (1994). 'Agenda-setting in the European Community', Journal of European Public Policy, 1.

Richardson, J.J. (1996). 'Eroding EU Policies: Implementation Gaps, Cheating and Re-Steering', in J.J. Richardson, ed., European Union: Power and Policy-Making. London: Routledge.

Risse-Kappen, T. (1991). 'Public Opinion, Domestic Structure and Foreign Policy in Liberal Democracies', World Politics, 43.

Rosenau, J. (1992). 'Governance, Order and Change in World Politics', in J. Rosenau \& E.O. Czempiel, eds., Governance without Government: Order and Change in World Politics. Cambridge: Cambridge University Press.

Sartori, G. (1996). Comparative Constitutional Engineering. London: Macmillan.

Sbragia, A. ed. (1992). Euro-Politics: Institutions and Policy-Making in the New European Community. Washington: The Brookings Institution. 
Steinmo, S. et al. (1992). Structuring Politics. Cambridge: Cambridge University Press.

Tsoukalis, L. (1997). The New European Economy, Third Edition. Oxford: Oxford University Press.

Weiler, J.H. (1991). 'The Transformation of Europe', Yale Law Journal, 100. Wendt, A. (1994a). 'Anarchy is What States Make of it: The Social Construction of Power Politics', International Organization, 48.

... (1994b). 'Collective Identity Formation and the International State' The American Political Science Review, 88. 